\title{
Avaliação da condição bucal dos pacientes em centro de terapia intensiva de um
}

\section{Hospital Metropolitano de Belo Horizonte}

\author{
Evaluation of the oral condition of patients in the intensive care center of a Metropolitan Hospital \\ in Belo Horizonte
}

Evaluación del estado bucal de pacientes en el centro de cuidados intensivos de un Hospital

Metropolitano de Belo Horizonte

Recebido: 20/08/2021 | Revisado: 28/08/2021 | Aceito: 30/08/2021 | Publicado: 02/09/2021

\author{
Júbila Aparecida Costa Reis \\ ORCID: https://orcid.org/0000-0002-1442-3659 \\ Hospital Metropolitano Odilon Behrens, Brasil \\ E-mail:jubilareis@hotmail.com \\ Júlia Cândido Leão \\ ORCID: https://orcid.org/0000-0001-6131-1003 \\ Hospital Metropolitano Odilon Behrens, Brasil \\ E-mail: juliacandidoleao@gmail.com \\ Tatiane Bretas da Silva Telles \\ ORCID: https://orcid.org/0000-0002-7264-5727 \\ Hospital Metropolitano Odilon Behrens, Brasil \\ E-mail: tati.bretas.telles@gmail.com \\ Cristina Oliveira Rodrigues \\ ORCID: https://orcid.org/0000-0003-0552-7330 \\ Hospital Metropolitano Odilon Behrens, Brasil \\ E-mail: crisolirodrigues@gmail.com \\ Hoberdan Oliveira Pereira \\ ORCID: https://orcid.org/0000-0002-7290-3677 \\ Hospital Metropolitano Odilon Behrens, Brasil \\ E-mail: hoberdanoliveira@gmail.com \\ Renata Gonçalves Resende \\ ORCID: https://orcid.org/0000-0001-7610-0399 \\ Faculdade Arnaldo Janssen, Brasil \\ Hospital Metropolitano Odilon Behrens, Brasil \\ E-mail: renatagresende@yahoo.com.br
}

\begin{abstract}
Resumo
O objetivo do estudo foi avaliar a condição bucal dos pacientes em Centro de Terapia Intensiva (CTI) de um hospital do município de Belo Horizonte, Minas Gerais. Trata-se de um estudo epidemiológico transversal, retrospectivo e descritivo de prontuários. Foram avaliados 112 prontuários, entre janeiro e dezembro de 2015. A maior parte dos pacientes eram homens $(53,57 \%)$, entre a $5^{\mathrm{a}}$ e a $6^{\mathrm{a}}$ décadas de vida $(25,89 \%)$. Os pacientes mais velhos, entre a $5^{\mathrm{a}}$ e a $9^{a}$ década de vida, apresentaram uma maior permanência no CTI. Casos de infecção por pneumonia foram observados em 40 pacientes $(35,71 \%)$, que também apresentou correlação com o aparecimento de alterações de mucosa bucal. A pneumonia nosocomial foi encontrada em 16 pacientes $(14,28 \%)$, sendo que nove eram desdentados parciais $(56,25 \%)$. Quando se analisou a série histórica da incidência de pneumonia associada a ventilação mecânica (PAVM) neste nosocômio, notou-se uma redução de 54,9\% comparando o ano de 2005, ano de introdução do serviço de odontologia hospitalar, ao ano de 2015. Esse estudo sugere que as alterações da cavidade bucal podem estar relacionadas às infecções hospitalares, como as pneumonias e ainda que a presença do cirurgião-dentista (CD) impacta positivamente o estado geral de saúde do paciente
\end{abstract}

Palavras-chave: Pneumonia associada a assistência à saúde; Unidade de Terapia Intensiva; Saúde bucal.

\section{Abstract}

The objective of the study was to evaluate the oral condition of patients in the Intensive Care Center (ICU) of a hospital in the city of Belo Horizonte, Minas Gerais. This is a cross-sectional, retrospective and descriptive epidemiological study of medical records. 112 medical records were evaluated between January and December 2015. Most patients were men (53.57\%), between the 5th and 6th decades of life $(25.89 \%)$. Older patients, between the 5th and the 9th decade of life, had a longer stay in the ICU. Cases of pneumonia infection were observed in 40 patients (35.71\%), which also correlated with the appearance of changes in the oral mucosa. Nosocomial pneumonia was found in 16 patients $(14.28 \%)$, nine of which were partially edentulous $(56.25 \%)$. When the historical series on the 
incidence of pneumonia associated with mechanical ventilation (VAP) was analyzed in this hospital, there was a reduction of 54.9\% comparing the year 2005, the year of introduction of the hospital dentistry service, to the year 2015. This study suggests that changes in the oral cavity may be related to nosocomial infections, such as pneumonia, and even though the presence of the dentist positively impacts the patient's general health.

Keywords: Respiratory infection; Intensive Therapy; Oral microbiota.

\section{Resumen}

El objetivo del estudio fue evaluar la condición bucal de pacientes en la Unidad de Cuidados Intensivos (UCI) de un hospital de la ciudad de Belo Horizonte, Minas Gerais. Se trata de un estudio epidemiológico transversal, retrospectivo y descriptivo de historias clínicas. Se evaluaron 112 historias clínicas entre enero y diciembre de 2015. La mayoría de los pacientes eran hombres $(53,57 \%)$, entre la $5^{\mathrm{a}}$ y la $6^{\mathrm{a}}$ década de la vida $(25,89 \%)$. Los pacientes mayores, entre la $5^{\mathrm{a}}$ y la $9^{\mathrm{a}}$ década de la vida, tenían una estancia más prolongada en la UCI. Se observaron casos de infección por neumonía en 40 pacientes $(35,71 \%)$, lo que también se correlacionó con la aparición de cambios en la mucosa oral. Se encontró neumonía nosocomial en 16 pacientes $(14,28 \%)$, nueve de los cuales eran parcialmente desdentados $(56,25 \%)$. Al analizar la serie histórica de incidencia de neumonía asociada a ventilador (NAV) en este hospital, se observó una reducción del 54,9\% comparando el año 2005, año de implantación del servicio de odontología hospitalaria, con el año 2015. Este estudio sugiere que los cambios en la cavidad bucal pueden estar relacionados con infecciones hospitalarias, como neumonía, y que la presencia de un dentista (DC) tiene un impacto positivo en el estado de salud general del paciente.

Palabras clave: Neumonía asociada a la atención médica; Unidad de Cuidados Intensivos; Salud bucal.

\section{Introdução}

No Brasil, até a década de 70, os pacientes internados eram acompanhados apenas pela equipe médica. Aqueles que apresentavam situações mais graves eram cuidados pelos enfermeiros em lugares não apropriados para tal tratamento. Após essa data, percebeu-se a necessidade de implantação dos Centros de Terapias Intensivas (CTIs) para melhor assistir aos pacientes, marcando assim, um grande progresso conquistado pelos hospitais (Schlesener, Rosa \& Raupp, 2012).

Hoje a equipe multidisciplinar é composta por vários profissionais da área da saúde e tem como objetivo discutir os casos e realizar as intervenções, em que, cada um na sua área específica, busca os melhores resultados na condição sistêmica do paciente (Alves, Ramos \& Penna, 2005). Isso porque há a importância da abordagem integral do ser humano, visto sua complexidade quando se encontra hospitalizado, mostrando a relevância da atuação conjunta dos diversos profissionais Dantas et al. (2015).

Já no século XIX surgiu na América a odontologia hospitalar com o objetivo de assistir ao paciente internado de forma integral e humanizada Dantas et al. (2015). Os cirurgião-dentista (CD), executam procedimentos de baixa, média ou alta complexidade, assim como os cuidados a higienização e alterações bucais, que são realizados em ambiente hospitalar, proporcionando uma melhora na saúde geral do paciente (Projeto de Lei nº 2776 (2008)).

No entanto, a presença dessa equipe multiprofissional, principalmente do $\mathrm{CD}$, não é uma realidade em todos os hospitais brasileiros, ainda que vários estudos comprovem o quanto a condição bucal influência no estado clínico do paciente (Jardim, Setti, Cheade \& Mendonça, 2013), (Sousa; Pereira \& Silva, 2014). A cavidade bucal requer higienização correta e específica e o CD tem o conhecimento sobre a microbiota presente, além das doenças que podem afetar e se relacionar com outra parte sistêmica do paciente Jardim et al. (2013). Além disso, o CD ainda tem capacidade de intervir em situações de emergência, diagnosticar doenças e interpretar exames (Jardim et al. 2013), (Morais et al. (2006). Sendo assim, a sua presença é de extrema relevância nas equipes multiprofissionais, para atuarem na educação, através de instruções quanto técnica de higienização correta e prevenção de doenças oportunistas presentes em toda área hospitalar, com a avaliação das alterações na mucosa, gengiva e dentes (Lima, Saliba, Garbin, Fernandes \& Garbin, 2011), Dantas et al. (2015).

A microbiota bucal dos pacientes saudáveis e dos internados em uma CTI são diferentes, devido à proliferação de patógenos com diferentes virulências e a perda da homeostasia do meio Dantas et al. (2015). Sabe-se que entre 48 e $72 \mathrm{~h}$ de internação em um CTI o indivíduo apresenta mudança em sua microbiota, sendo colonizado por patógenos gram-negativos e 
anaeróbicos presentes no acúmulo de biofilme que são agentes etiológicos de doenças periodontais e doenças respiratórias, como a pneumonia nosocomiais Dantas et al. (2015), Portaria SES-DF №287 (2016). Essa infecção hospitalar está associada a utilização de ventilação mecânica, sendo considerada pneumonia associada à ventilação mecânica (PAVM) desenvolvida a partir de $48 \mathrm{~h}$ a partir do início da ventilação mecânica, tendo sua instalação pela aspiração dos microrganismos somada ao estado debilitado do paciente, não sendo diagnosticada no momento da internação Lima et al. (2011), (Gomes \& Esteves, 2012), (Souza, 2016). Pela virulência desses patógenos são desenvolvidas várias outras doenças sistêmicas, como: endocardite infecciosa, cardiovasculares, acidentes vasculares encefálicos, infecções de próteses articulares, aterosclerose e infarto agudo do miocárdio (Souza, 2016). Nesse sentido, os pacientes internados nos CTIs precisam de muita atenção da equipe odontológica, principalmente aqueles com ventilação mecânica e traqueostomia, para instalação do protocolo de higienização da cavidade bucal com ações de limpeza dentária, gengivas, mucosas, tubo orotraqueal e qualquer outro dispositivo presente na cavidade oral, além de tratar diversos processo patológicos que acometem a cavidade bucal (Alves, Ramos \& Penna, 2005), Lima et al. (2011). A clorexidina $0,12 \%$, é até o momento, o mais eficaz no controle do biofilme dental, por apresentar boa substantividade e efeito bacteriostático (Lotufo, Solis \& Pannuti, 2005).

Sendo assim, a presença da equipe multidisciplinar no ambiente hospitalar e nos CTIs é de fundamental importância e visa a excelência no cuidado ao paciente. É sabido que cada profissional tem seu papel específico dentro do planejamento global de abordagem ao paciente. Nesse sentido, a presença do profissional CD no CTI visa ao atendimento integral ao paciente, minimizando os agravos decorrentes da presença de patologias bucais. Já é sabido que há uma ligação entre a cavidade bucal e a saúde sistêmica. Sendo assim, quando o paciente se encontra em uma UTI a condição bucal dele pode interferir em sua condição sistêmica, ocasionando assim grande prejuízo no que se refere à melhora clínica do paciente. Baseado no exposto, o objetivo desse trabalho é analisar o perfil de condição bucal dos pacientes em CTI do Hospital Metropolitano Odilon Behrens (HMOB), no município de Belo Horizonte, Minas Gerais.

\section{Metodologia}

Trata-se de um estudo epidemiológico transversal, fundamentado na análise retrospectiva e descritiva de prontuários, com abordagem quantitativa dos dados, aprovado pelo Comitê de Ética em Pesquisa (CEP) do HMOB e pela Comissão Nacional de Ética em Pesquisa (CONEP) nº 2.343.761.

Foram analisados prontuários de pacientes hospitalizados nos Centros de Terapia Intensiva (CTIs) do HMOB e avaliados pelo Serviço de Odontologia Hospitalar deste nosocômio, no período de janeiro a dezembro de 2015, atendendo a critério de conveniência, referentes a completude ou disponibilidade de dados.

Foram coletados dados referentes ao perfil demográfico dos indivíduos internados em CTI do HMOB, presença de registro quanto à realização da higiene bucal pela equipe de enfermagem assistente, alterações bucais mais prevalentes nesses pacientes, tipo de internação, causas de óbitos, tempo permanência no CTI, doenças secundárias e infecções oportunistas.

A análise estatística dos dados foi realizada por meio do programa Statistical Packag for the Social Sciences (SPSS), versão 23.0 (SPSS Inc., USA). Os resultados obtidos no estudo foram expressos por valores absolutos, médias, frequências, percentuais e chi-quadrado.

\section{Resultados}

Foram selecionados 262 prontuários, referentes aos meses de janeiro a dezembro de 2015. Destes, 150 foram descartados por registros incompletos. Foram incluídos nesta pesquisa 112 prontuários. A média de idade no momento da internação foi de 60,88 anos para homens e 65,76 anos para mulheres.

Observou-se uma maior prevalência de internação em CTI de pacientes do sexo masculino (53,57\%), com a sua maior 
frequência entre a $5^{\mathrm{a}}$ e $6^{\mathrm{a}}$ décadas de vida $(25,89 \%)$, conforme mostra a tabela 1 . Foi possível notar ainda que quanto mais velho o indivíduo, entre a $5^{\text {a }}$ e a $9^{\text {a }}$ década de vida $(79,45 \%$ do total avaliado), maior o seu tempo de internação (internação maior que 30 dias, correspondendo a 46,60\% dos casos), ao passo que indivíduos entre a $1^{\text {a }}$ e $4^{\text {a }}$ décadas de vida representaram 20,54\% da amostra e permaneceram no CTI pelo período máximo de 30 dias (15,17\%) (Dados não mostrados).

Tabela 1 - Distribuição dos pacientes por sexo e faixa etária (n=112).

\begin{tabular}{lccccc}
\hline Sexo & \multicolumn{3}{c}{ Faixa } & \multicolumn{1}{c}{ etária } & \\
\hline & $1^{a} / 2^{a}$ & $3^{a} / 4^{a}$ & $5^{a} / 6^{a}$ & $7^{a} / 8^{a} / 9^{a}$ & \\
& Décadas & Décadas & Décadas & Décadas & Total \\
Masculino & $4(3,57 \%)$ & $8(7,14 \%)$ & $29(25,89 \%)$ & $19(16,96 \%)$ & $60(53,57 \%)$ \\
Feminino & $1(0,89 \%)$ & $10(8,92 \%)$ & $16(14,28 \%)$ & $25(22,32 \%)$ & $52(46,42 \%)$ \\
Total & & & & & \\
& $\mathbf{5 ( 4 , 4 6 \% )}$ & $\mathbf{1 8}(\mathbf{1 6 , 0 7 \% )}$ & $\mathbf{4 5}(\mathbf{4 0 , 1 7 \% )}$ & $\mathbf{4 5}(\mathbf{3 9 , 2 8 \% )}$ & $\mathbf{1 1 2 ( 1 0 0 \% )}$ \\
\hline
\end{tabular}

Fonte: Autores.

Quanto às pneumonias, foram diagnosticados 40 casos da doença $(37,71 \%)$, sendo que em 17 pacientes $(15,17 \%)$ ocorreu entre o dia 0 e dia 30; 22 (55\%) permaneceram no CTI em um período de 30 a 90 dias e apenas 1 paciente desenvolveu a doença com o período de internação maior que 90 anos (Dados não mostrados). Além do tempo de permanência do paciente no CTI, as alterações na semi-mucosa labial, como ressecamento labial, úlceras e descamação, foram observadas em 21 doentes $(52,50 \%)$ e estas também estavam relacionadas com o aparecimento de outros tipos de pneumonias (Tabela 2).

A Tabela 2 mostra ainda correlação positiva entre pacientes acometidos por pneumonia, 16 pacientes $(14,28 \%)$, e a ocorrência de pneumonia nosocomial (PN), sendo que destes houve predominância na $5^{\mathrm{a}}$ e $6^{\mathrm{a}}$ década de vida, correspondendo a 8 destes indivíduos (50\%) (Tabela 2). E ainda, destes indivíduos acometidos com a PN, foi constatado que 9 deles eram desdentados parciais $(56,25 \%)$.

Tabela 2 - Correlação entre faixa etária e perfil de dentição dos pacientes em CTI com a ocorrência de pneumonia nosocomial $(\mathrm{n}=112)$.

\begin{tabular}{|c|c|c|c|}
\hline Variável & Pneumonia & nosocomial & p* \\
\hline Faixa etária & Não & Sim & \\
\hline $1^{\mathrm{a}} / 2^{\mathrm{a}}$ décadas & $3(2,67 \%)$ & $2(1,78 \%)$ & 0,018 \\
\hline $3^{\mathrm{a}} / 4^{\mathrm{a}}$ décadas & $15(13,39 \%)$ & $3(2,67 \%)$ & \\
\hline $5^{\mathrm{a}} / 6^{\mathrm{a}}$ décadas & $37(33,03 \%)$ & $8(7,14 \%)$ & \\
\hline $7^{\mathrm{a}} / 8^{\mathrm{a}} / 9^{\mathrm{a}}$ décadas & $41(36,60 \%)$ & $3(2,67 \%)$ & \\
\hline Dentição & Não & Sim & \\
\hline Completa & $17(15,45 \%)$ & $5(4,54 \%)$ & \\
\hline Desdentado parcial & $35(31,81 \%)$ & $9(8,18 \%)$ & 0,043 \\
\hline Desdentado total & $42(38,18 \%)$ & $2(1,81 \%)$ & \\
\hline Alterações de semi-mисоsa labial & Não & Sim & \\
\hline Sem alterações & $55(49,54 \%)$ & $18(16,21 \%)$ & \\
\hline Alterada $^{\#}$ & $15(13,51 \%)$ & $21(18,91 \%)$ & 0,006 \\
\hline Não foi possível o exame & $1(0,90 \%)$ & $1(0,90 \%)$ & \\
\hline
\end{tabular}

\# ressecamento labial, úlceras e descamação. *Teste estatístico: qui-quadrado. Fonte: Autores.

Além disso, quando se analisa a série histórica os dados brutos fornecidos pela Comissão de Controle de Infecção 
Relacionada à Assistência à Saúde (CCIRAS-HMOB), observou-se uma redução de 54,10\% na ocorrência de PAVM quando se compara o ano de 2005, ano que o serviço de odontologia hospitalar iniciou suas atividades, ao de 2015, quando o profissional da odontologia já apresentava 10 anos de inserção na rotina diária dos CTIs (Tabela 3).

Tabela 3 - Distribuição por época na ocorrência de Pneumonia Associada a Ventilação Mecânica.

\begin{tabular}{lc}
\hline Época (anos) & Densidade de incidência de PAVM \\
\hline 2005 & $14,6 \%$ \\
2015 & $7,9 \%$ \\
\end{tabular}

Fonte: CCIRAS HMOB (2018).

\section{Discussão}

O HMOB é um dos poucos hospitais mineiros que tem uma equipe multidisciplinar com a presença do CD dentro dos seus três CTIs, há mais de 10 anos. O CD, nessa unidade, realiza avaliações, instruções de higienizações aos técnicos de enfermagem e intervenções na cavidade bucal, sendo que essas intervenções são de drenagem de abscesso, exodontias e raspagens de cálculos. Na avaliação da cavidade bucal são observadas as alterações de lábios, mucosa extra e intrabucal, dentes, palato duro e mole, assoalho bucal e língua, além da dentição e condição de higiene bucal do paciente. Nesse sentido, os resultados deste trabalho mostram, de forma pioneira, que a presença do CD apresenta extrema relevância dentro dos CTIs, no que se refere a redução de infecções hospitalares como a PN, o que é corroborado por diversos estudos Schlesener et al. (2012); Dantas et al. (2015); Jardim et al. (2013); (Gomes \& Esteves, 2012); (Camargos \& Gonzaga, 2015).

Este estudo mostrou um maior número de pacientes do sexo masculino, sendo a maioria deles entre 50 e 60 anos, ao passo que as mulheres hospitalizadas em CTI concentram-se em faixas etárias mais avançadas. Mulheres mais velhas na condição de internação pode ser explicado pelo fato de que esse sexo apresenta uma maior expectativa de vida, maiores cuidados com saúde, já que os homens apresentam adoecimento mais severo em faixas etárias mais precoces (Camargos \& Gonzaga, 2015); (Ewers, Rizzo \& Filho, 2008).

O tempo médio de permanência dos pacientes no CTI, que se mostrou mais relevante neste trabalho, foi de até 30 dias. Não foi observado um consenso na literatura sobre o período que os pacientes permanecem em CTIs, o que pode ser explicado pelo fato da maioria dos estudos serem realizados em populações mistas, isto é, pacientes hospitalizados por perfis diversos como clínica médica e clínica cirúrgica Oliveira et al. (2010).

A pneumonia é a segunda infecção que mais acomete pacientes em leito de CTI Morais et al. (2006); Oliveira et al. (2010); Souza et al. (2017); (Oliveira, Carneiro, Fscherl \& Tinoco, 2007). O resultado dessa pesquisa mostra que, dos pacientes acometidos por essa doença, mais da metade tiveram sua permanência prolongada dentro dos cuidados intensivos, entre 30 e 90 dias, sugerindo que quanto mais tempo a permanência no ambiente hospital, maior propensão a infecções oportunistas, como a pneumonia. Esse mesmo achado é relatado pelo trabalho de Souza et al Souza et al. (2017), que mostra que o quadro clínico do paciente pode ser agravado pela ocorrência de pneumonia levando a um aumento do período de internação ou até mesmo ao óbito do indivíduo.

Nos pacientes que apresentaram pneumonias, mais da metade apresentaram alguma alteração de mucosa bucal. O paciente hospitalizado muitas vezes faz uso de diversos dispositivos de suporte à vida, tais como de tubo orotraqueal, ou medicações, o que levam a cavidade bucal a permanecer aberta ou semi-aberta por longo período de tempo Morais et al. (2006); (Camargos \& Gonzaga, 2015); Oliveira et al. (2007). A necessidade da utilização suporte respiratório avançado pode 
causar ressecamento nos lábios e na mucosa intrabucal do indivíduo, favorecendo a proliferação dos patógenos da cavidade bucal, e a sua aspiração para o pulmão, causando doenças respiratórias (Camargos \& Gonzaga, 2015); (Cruz, Morais \& Trevisani, 2014). Essa pesquisa mostra que a maioria das alterações de mucosas ocorridas nos pacientes foram ressecamento labial e em outros momentos notou-se ulceração e descamação. Traçar uma linearidade entre esses eventos pode ainda ser precoce, tendo em vista a escassez de estudos, no entanto esses achados bucais alertam para a importância dos cuidados com higiene e hidratação bucal.

Este estudo mostrou ainda que a população mais idosa foi a mais acometida por pneumonia nosocomial. Essa pneumonia requer atenção especial da equipe multidisciplinar, devido à alta morbidade e mortalidade Schlesenerel et al. (2012); Cruz et al. (2014). Dos 16 pacientes acometidos pela doença, 10 vieram a óbito. Estudos mostram que os pacientes mais idosos têm uma piora na resposta imunológica, que é denominado imunosenescência, o que pode explicar o prognóstico ruim nesse perfil de pacientes (Camargos \& Gonzaga, 2015); (Rabelo, Queiroz \& Santos, 2010). Gomes et al. (Gomes \& Esteves, 2012), também verificaram em seu estudo a relação dos pacientes mais idosos adquirirem pneumonia nosocomial.

Além do ambiente ser um fator de propensão ao acúmulo de microrganismos, a própria cavidade bucal apresenta áreas de retenção de patógenos, como as superfícies de dentes e próteses fixas ou removíveis Dantas et al. (2015); Cruz et al. (2014); (Pinheiro \& Almeida, 2014); Batista et al. (2014). Já é sabido que a presença dos microrganismos nas superfícies bucais é um fator etiológico de um tipo específico de pneumonia, a nosocomial Cruz et al. (2014). Mais da metade dos pacientes acometidos com essa pneumonia eram desdentados parciais, mostrando uma provável presença de superfícies dentais e protéticas para o acúmulo de biofilme. O biofilme apresenta patógenos bucais e respiratórios, que pela presença do tubo orotraqueal colonizam as vias respiratórias superiores causando a pneumonia nosocomial Oliveira et al. (2010); Cruz et al. (2014).

De todos os pacientes relatados com pneumonia nosocomial, 10 deles apresentaram alterações linguais, sugerindo que a presença da saburra é um fator determinante para a doença. Esse achado também é encontrado no estudo de Cruz et al. Cruz et al. (2014), em que é encontrado a presença de saburra lingual estando associada aos patógenos causadores da pneumonia nosocomial3. A melhor solução para a redução da saburra lingual é a higienização da cavidade bucal.

Já é consenso que a presença do CD dentro das unidades hospitalares e dos CTIs é uma grande benfeitoria devido à redução nas infecções Lima et al. (2011); Ewers et al. (2008); Oliveira et al. (2010). Isso foi também confirmado por esta pesquisa, que evidenciou uma significativa redução de PAVM, em um intervalo de 10 anos desde a introdução da odontologia nos CTIs, mostrando, portanto, a excelência na atuação do CD dentro desta unidade. Sendo assim, o paciente se beneficia com assistência integral e o hospital tem uma redução importante de custos no que se refere aos cuidados ao paciente crítico, já há uma redução no período de internação e diminuição do uso de medicações Lima et al. (2011); (Rocha \& Ferreira, 2014); (Rabelo, Queiroz \& Santos, 2010).

\section{Conclusão}

Este trabalho mostrou a importância da presença do CD dentro da equipe multidisciplinar e evidenciou que as alterações da cavidade bucal podem estar relacionadas às infecções hospitalares, como as pneumonias, e ainda que a presença do profissional da odontologia impacta positivamente o estado geral de saúde do paciente.

Nesse sentido, estudos futuros com amostras ampliadas e análises prospectivas são necessárias para confirmar os resultados sugeridos nessa pesquisa e ampliar o entendimento sobre a importância da odontologia hospitalar.

\section{Referências}


Alves, M., Ramos, F. R. S., \& Penna, C. M. M. (2005). O trabalho interdisciplinar: aproximações possíveis na visão de enfermeiras de uma unidade de emergência. Contexto Enferm., 14(3): 31-323.

Batista, A. S., Siqueira, J. S. S., Jr Silva, A., Ferreira, M. F., Agostini, M., \& Torres, S. R. (2014). Alterações orais em pacientes internados em unidades de terapia intensiva. Rev. bras. odontol., 71(2): 9-156.

Camargos, M. C. S., \& Gonzaga, M. R. (2015). Viver mais e melhor? Estimativas de expectativa de vida saudável para a população brasileira. Cad. Saúde Pública, 31(7): 1460-1472.

Cruz, M. K., Morais, T. M. N., \& Trevisani, D. M. (2014). Avaliação clínica da cavidade bucal de pacientes internados em unidade de terapia intensiva de um hospital de emergência. Revista Bras Ter Intensiva, 26(4): 379-383.

Dantas, B. O., Araujo, I. A., Araujo, H. B. N., Araujo, E. C., Bezerra, A. C. B., \& Miranda, A. F. (2015). Saúde bucal e cuidados na Unidade de Terapia Intensiva. Revista Odontol Planal Cent., 5(1): 28-32.

Ewers, I., Rizzo, L. V., \& Filho, J. K. (2008). Imunologia e envelhecimento Einstein, 6(1), 13-20.

Gomes, S. F., \& Esteves, M. C. L. (2012). Atuação do cirurgião-dentista na UTI: um novo paradigma. Revista Bras Odontol., 69(1): 67-70.

Jardim, E. G., Setti, J. S., Cheade, M. F. M., \& Mendonça, J. C. G. (2013). Atenção odontológica a paาcientes hospitalizados: Revisão da literatura e proposta de protocolo de higiene oral. Revista Bras Ciên Saúde., 11(35): 31-36.

Lima, D. C., Saliba, N. A., Garbin, A. J. I., Fernandes, L. A., \& Garbin, C. A. S. (2011). A importância da saúde bucal na ótica de pacientes hospitalizados: Revista Ciência \& Saúde Coletiva., 16(1):1173-1180.

Lotufo, R. F. M., Solis, A. C. O., \& Pannuti, C. M. (2005). Bases racionais para indicação de antimicrobianos locais e sistêmicos em Periodontia. Atualização Clínica em Odontologia, Anais do Congresso Internacional de Odontologia de São Paulo. 381-393.

Morais, T. M. N., Silva, A., Avi, A. L. R. O., Souza, P. H. R., Knobel, E., \& Camargo, L. F. A. (2006). A Importância da Atuação Odontológica em Pacientes Internados em Unidade de Terapia Intensiva. Revista Brasileira de Terapia Intensiva, 18(4).

Oliveira, A. B. F., Dias, O. M., Mello, M. M., Araújo, S., Dragosavac, D., \& Nucci, A. (2010). Factors associated with increased mortality and prolonged length of stay in an adult intensive care unit. Rev Bras Ter Intensiva, 22(3), 6-250.

Oliveira, L. C. B. S., Carneiro, P. P. M., Fscherl, R. G., \& Tinoco, B. E. M. (2007). A presença de patógenos respiratórios no biofilme bucal de pacientes com pneumonia nosocomial. Revista Brasileira de Terapia Intensiva, 19(4): 428-433.

Pinheiro, T. S., \& Almeida, T. F. (2014). A saúde bucal em pacientes em UTI. Revista Bahiana de Odontologia, 5(2): 94-103.

Portaria SES-DF Nº287 de 02 de dezembro de 2016, Atendimento Odontológico em UTI, publicada no DODF Nº 228 de 06.12.2016.

Projeto de Lei $\mathrm{n}^{\circ}$ 2776/08 da Câmara dos Deputados que estabelece a obrigatoriedade da presença de profissionais de odontologia nas unidades de terapia intensiva e dá outras providências.

Rabelo, G. D., Queiroz, C. I., \& Santos, P. S. S. (2010). Atendimento odontológico ao paciente em unidade de terapia intensiva. Arq Med Hosp Cienc Med Santa Casa São Paulo, 55(2): 67-70

Rocha, L. M., \& Ferreira, E. F. (2014). Odontologia hospitalar: a atuação do cirurgião dentista em equipe multiprofissional na atenção terciária. Arq Odontol, Belo Horizonte, 50(4): 154-160.

Schlesener, V. R. F., Rosa, U. D., \& Raupp, S. M. M. (2012). O cuidado com a saúde bucal de pacientes em UTI. Cinergis., 13(1): 73-77.

Sousa, L. V. S.; Pereira, A. F. V., \& Silva, N. B. S. (2014). A atuação do cirurgião-dentista no atendimento hospitalar. Rev Ciências da Saúde., 16(1): 39-45.

Souza, A. F. (2016) Identificação de condições de saúde bucal em pacientes submetidos à cirurgia cardíaca: monitoramento de prováveis fatores de risco para endorcadite. 111f. Dissertação (Mestrado) - Faculdade de Odontologia, Universidade Federal de Minas Gerais, Belo Horizonte.

Souza, L. C. D., Mota, V. B. R., Carvalho, A. V. S. Z., Corrêa, R. G. C. F., Libério, A. S., \& Lopes, F. F. (2017). Association between pathogens from tracheal aspirate and oral biofilm of patients on mechanical ventilation. Braz. Res oral, 31: 1-18. 